\title{
Alfons Siegel \\ Völkerbund, Hexagon und die Zukunft der UNO Kant-Bezüge in Friedenskonzepten von Matthias Erzberger und Dieter Senghaas
}

\section{Vorbemerkung}

Was soll eine vergleichende Betrachtung zu Kant-Bezügen bei einem prominenten Zentrumspolitiker im ersten Drittel des 20. Jahrhunderts ${ }^{1}$ mit denen bei einem international renommierten Sozialwissenschaftler der Gegenwart? Manchen mag bekannt sein, dass Matthias Erzberger als Leiter der deutschen Waffenstillstandskommission mit seiner Unterschrift im November 1918 zum Ende des Ersten Weltkrieges beitrug und wohl auch deswegen drei Jahre später von irregeleiteten Fanatikern ermordet wurde. Der Friedens- und Konfliktforscher Dieter Senghaas ist sicher vielen einschlägig Interessierten durch seine Kritik der Abschreckungspolitik, die Studien zur Entwicklungsforschung oder auch wegen seiner konstruktiven Vorschläge zur Friedensgestaltung mit dem Ende des Ost-West-Konfliktes vertraut. Aber reicht dies schon als Grund und Stoff für eine vergleichende Reflexion? Plausibler wird ein solches Vorhaben allerdings, wenn man sich klarmacht, dass sich Erzberger eben nicht schon mit der Beendigung des Krieges im Sinne eines Schweigens der Waffen zufrieden geben wollte, sondern dass insbesondere sein 1918 publiziertes Buch zur Problematik eines Völkerbundes ${ }^{2}$ dazu gedacht war, einen dauerhaften Weltfrieden anzubahnen. Damit aber entspricht Erzbergers politisches Grundmotiv prinzipiell jenem von Dieter Senghaas, dem es als Wissenschaftler darum geht, Voraussetzungen bzw. Strukturbedingungen dauerhaften Friedens ebenfalls bis in die globale Ebene hinein systematisch zu konzeptualisieren.

Im vorliegenden Beitrag geht es jedoch nicht um eine umfassende Darstellung der friedenskonzeptionellen Reaktionen beider Autoren auf die historischen Umbrüche ihrer Epochen, ${ }^{3}$ sondern um eine vertiefende Betrachtung darüber, wie bei Erzberger und Senghaas ganz spezifisch dabei die Friedensphilosophie Immanuel Kants herangezogen und verarbeitet wird. Erzbergers Bezugnahme zeigt sich bereits äuBerlich darin, dass er auf Kants Völkerbund-Idee aufbaut. Senghaas dokumentiert

1 Eine relativ breite Grundinformation bieten neuerdings: Christoph E. Palmer / Thomas Schnabel (Hg.), Matthias Erzberger. 1875-1921. Patriot und Visionär, Stuttgart/Leipzig 2007.

2 Matthias Erzberger, Der Völkerbund. Der Weg zum Weltfrieden, Berlin 1918.

$3 \mathrm{Vgl}$. Alfons Siegel, Ideen zur Friedensgestaltung am Ende des Ersten Weltkrieges und des Ost-West-Konfliktes. Entwicklungen und Konzepte von Matthias Erzberger und Dieter Senghaas, Münster 2003. 
sein Stehen bzw. Fortschreiten in der Kant'schen Denktradition demonstrativ mit dem Titel des 2004 - im 200. Todesjahr des großen Philosophen - publizierten Buches Zum irdischen Frieden, ${ }^{4}$ das somit nicht zufällig eine Analogie zu Kants berühmter Schrift Zum ewigen Frieden ${ }^{5}$ von 1795 erkennen lässt und auch im Grobaufbau eine bewusst gewählte Parallelstruktur aufweist. Senghaas' Buch darf somit als Versuch verstanden werden, für »das Kantische Projekt eine weltkundige zeitgemäße Konkretion « anzubieten. ${ }^{6}$

Mit den angerissenen und deutlicher herauszuarbeitenden Entsprechungen, aber auch mit zutage tretenden Divergenzen der verschiedenen Friedensdenker wird überschaubar und transparenter, was an den friedenskonzeptionellen Entwürfen aus drei Epochen (dem Aufklärungszeitalter, dem Ende des Ersten Weltkrieges und dem Ende des Kalten Krieges) zeitlos gültig bleibt, inwiefern sie einer veränderungsbedingten Korrektur, Ergänzung und Weiterentwicklung bedürfen. Der Beitrag ist in drei Hauptabschnitte gegliedert: In den ersten beiden wird zunächst Kant-Bezügen bei Erzberger und Senghaas separat nachgegangen, um im dritten, dem abschließenden, eine vergleichende Bilanz samt friedenspolitischer Schlussfolgerungen für die Gegenwart zu ziehen. Diese berühren bzw. inspirieren auch Gedanken zur Reform der UNO als Nachfolgeorganisation des Völkerbundes.

\section{Erzberger und Immanuel Kant}

\section{a) Bedeutung Kants für Erzbergers Friedensdenken und markante Grundverbindungen}

Dass die Philosophie Immanuel Kants eine nennenswerte Bedeutung für das friedenskonzeptionelle Denken von Matthias Erzberger hatte, lässt sich exemplarisch am Zitat einer Rede aufzeigen, die der mit dem Ende des Ersten Weltkrieges zum Staatssekretär (Kabinettsmitglied analog eines heutigen Ministers) aufgestiegene Zentrumspolitiker im Dezember 1918 in Berlin hielt. Erzberger führte dabei u.a. aus:

»Was Kant in seinen vielen Schriften zum Ausdruck brachte, soll heute im Völkerbund verwirklicht werden. Wer Kant heute liest, der wird glauben, Kant habe seine denkwürdigen Werke unter dem Eindruck des heutigen Menschheitselends geschrieben. $\ll^{7}$

4 Dieter Senghaas, Zum irdischen Frieden. Erkenntnisse und Vermutungen, Frankfurt am Main 2004.

5 Immanuel Kant, Zum ewigen Frieden. Ein philosophischer Entwurf. Herausgegeben von Rudolf Malter, Stuttgart 1984.

6 Vgl. Senghaas, Zum irdischen Frieden..., aaO. (FN 4), Text auf dem Buchrücken. Mit dem Begriffsgebrauch »weltkundig« erinnert Senghaas ebenfalls an Kants Zum ewigen Frieden, wo einleitend vom »weltkundigen Staatsmann« die Rede ist; vgl. Immanuel Kant, Zum ewigen Frieden..., aaO. (FN 5), S. 3.

7 Matthias Erzberger, Der Völkerbund als Friedensfrage, Berlin 1919, S. 9. 
Bereits in Erzbergers rund ein Vierteljahr zuvor publiziertem Buch Der Völkerbund. Der Weg zum Weltfrieden hatte er sich ausführlicher auf verschiedene Schriften des Königsberger Aufklärers bezogen, ${ }^{8}$ um das Konzept einer Weltorganisation zu propagieren, mit der »die Wiederkehr eines ... Weltkrieges« verhütet werden sollte. ${ }^{9}$ Damit zählte der schwäbische Zentrumsmann zum internationalen Kreis jener Persönlichkeiten, die »das Kantische Projekt zum ersten Mal auf die Tagesordnung der praktischen Politik « brachten. ${ }^{10}$

Nicht nur sein friedenspolitisches Programm eines Völkerbundes, das sich in wesentlichen Details von der in den folgenden Jahren realisierten Version unterschied, ${ }^{11}$ begründete Erzberger nachdrücklich u.a. mit Kants Völkerbund-Idee, auch die Kritik an den von Erzberger diagnostizierten Kriegsursachen sowie Einzelgedanken zu deren Überwindung lassen sich mit ausdrücklichen Bezugnahmen auf Schriften Kants in Verbindung bringen.

Die allgemein mit Kant verbundenen Teilbereiche im friedenskonzeptionellen Denken Erzbergers können vorgreifend in zwei Hauptsträngen gebündelt werden:

- Seine Kritik herkömmlicher Gleichgewichtsprogrammatik hinsichtlich der Beziehungen zwischen Nationalstaaten und deren damit riskierte militärische Aufrüstung,

- seine Vorschläge zur Rüstungsbegrenzung, Verrechtlichung internationaler Beziehungen und einer Beteiligung der Völker im Sinne republikanisch bzw. demokratisch verfasster sowie föderativ zusammengeschlossener Rechtsstaaten.

Zur Unterscheidung von früheren Autoren, die ebenfalls schon Wegweisendes zur Herausforderung des Friedens geschrieben hatten, betont Erzberger mit Blick auf Kant, dass keiner von ihnen ${ }^{12}$ wie dieser den ewigen Frieden als ein sittliches Pflichtgebot aufgefasst « habe. ${ }^{13}$ Damit korrespondiert möglicherweise auch die Tatsache, dass Erzberger in seinem Völkerbund- und Friedenskonzept der Bemühung um weitgehende Integration von Moral und Politik ein besonderes Gewicht gibt. ${ }^{14}$

\section{b) Kontext Erzberger'scher Kant-Rezeption mit Übersicht entsprechender Schriften}

In seinem Buch Der Völkerbund. Der Weg zum Weltfrieden beschäftigt sich Erzberger im zweiten Teil des Kapitels »IV. Deutschland und der Weltfriede« zusam-

8 Vgl. Erzberger, Der Völkerbund. Der Weg zum Weltfrieden, aaO. (FN 2), S. 66-73.

9 Vgl. ebd., S. 5.

10 Vgl. Jürgen Habermas, Der gespaltene Westen. Kleine Politische Schriften X, Frankfurt am Main 2004, S. 154 (Kapitel 8.II.3. Kant, Woodrow Wilson und der Völkerbund).

11 Vgl. Siegel, Ideen zur Friedensgestaltung..., aaO. (FN 3), S. 122-152, insbes. S. 140-148.

12 Erzberger nennt als Beispiele J. F. von Palthen, Projekt einen immerwährenden Frieden zu unterhalten, den Völkerrechtler Vattel mit seinem Lehrbuch zur Frage des Schiedsgerichtes, den Abbé St. Pierre mit dem Projet de la paix perpétuelle, Jean Jacques Rousseau mit seinem Jugement sur la perpétuelle sowie die Engländer Swift und Hume; vgl. Erzberger, Der Völkerbund. Der Weg zum Weltfrieden, aaO. (FN 2), S. 66.

13 Vgl. ebd. (Hervorhebung im Original).

14 Vgl. Erzberger, Der Völkerbund als Friedensfrage, aaO. (FN 7), S. $5 f$. 
menhängend (über rund sechs der 196 Seiten umfassenden Publikation) mit Kant'scher Friedensphilosophie, nachdem er im ersten Teil dieses Buchkapitels der Tradition des Schiedsgerichtsgedankens in der deutschen Rechtsgeschichte nachgegangen war. In seiner Darstellung friedenstheoretisch relevanter Ideenbestände bei Kant stützen sich Erzbergers Ausführungen auf folgende sechs Schriften, wobei er sich ihrer in der Reihenfolge der Erscheinungsjahre bedient und Kants berühmtem Traktat Zum ewigen Frieden den meisten Platz (rund ein Drittel) einräumt:

- Idee zu einer allgemeinen Geschichte in weltbürgerlicher Absicht (1784),

- Muthmaßlicher Anfang der Menschengeschichte (1786),

- Über den Gemeinspruch: Das mag in der Theorie richtig sein, taugt aber nicht für die Praxis (1793),

- Zum ewigen Frieden. Ein philosophischer Entwurf (1795),

- Die Metaphysik der Sitten, (Rechtslehre) (1797),

- Der Streit der Fakultäten (1797).

Es handelt sich damit bei den von Erzberger herangezogenen Texten überwiegend um die sogenannten kleinen Schriften Kants, deren »einfache und pauschale Einordnung als >Begleitmusik< und >Nebenmelodie< mehr als fragwürdig «, der spezifischen »Bedeutung dieser Schriften « keineswegs adäquat wäre. ${ }^{15}$ Nachfolgend wird zumindest vermutbar, dass sich der Politiker mit diesem beachtlichen Spektrum friedensrelevanter Texte des Philosophen mehr als nur punktuell beschäftigt hat.

\section{c) Kants Geschichtsphilosophie - Impuls für Erzbergers Kritik von Überrüstung und Krieg}

Von Kants etwa 17 Seiten umfassender Abhandlung, Idee zu einer allgemeinen Geschichte in weltbürgerlicher Absicht, in der es um die Frage »nach dem Selbstverständnis des Individuums und dessen Verantwortung und Beitrag zu einer künftigen Idealordnung « geht ${ }^{16}$, auch als »geschichtsphilosophischer Prolog zur Friedenschrift « bezeichnet ${ }^{17}$ und in neun »Sätze« gegliedert, zitiert Erzberger eine längere Passage aus dem siebenten sowie eine kürzere aus dem achten Satz. »Die Natur« treibe, wie Erzberger Kants siebenten Satz ausschnitthaft und vereinfachend wiedergibt, »durch die Kriege, durch die überspannte und niemals nachlassende Zurüstung zu denselben ... aus dem gesetzlosen Zustande der Wilden hinauszugehen und in einen Völkerbund zu treten, wo jeder ... Staat seine Sicherheit und Rechte ... al-

15 Vgl. Theo Stammen, »Die Stellung der kleinen Schriften im >Kosmos des Wissens« in: ders. (Hg.), Kant als politischer Schriftsteller, Würzburg 1999 - künftig: Stammen, aaO. (FN 15) - S. 15f.

16 Vgl. Carolin Drube, "Idee zu einer Geschichte in weltbürgerlicher Absicht. Eine geschichtsphilosophische Abhandlung zwischen Zeitkritik und Zukunftsvision« in: Stammen, aaO. (FN 15), S. 59.

17 Vgl. Lothar Zahn, »Kant und die Problematik des neuesten Friedensbegriffs « in: Autorenteam der Pädagogischen Hochschule Schwäbisch Gmünd (Hg.), Frieden im Spektrum der Wissenschaften. Anregungen zu einer Friedenserziehung, Heidenheim 1973, S. 31. 
lein von diesem ... erwarten könnte. «18 Wenngleich diese Idee als schwärmerisch verlacht worden sei, wie Kant weiter zitiert wird, gehe es in Fortsetzung des Zwangs, die »brutale Freiheit« des wilden Menschen »in einer gesetzmäßigen Verfassung " gewissermaßen zu zivilisieren und letztlich über alle Kriege hinweg (als Versuche der Zerstückelung und Neubildung einzelstaatlicher Gebilde) »eine gemeinschaftliche Verabredung und Gesetzgebung «, also einen "Zustand « zu schaffen, »der, einem bürgerlichen gemeinen Wesen ähnlich, so wie ein Automat sich selbst erhalten « könne. ${ }^{19}$ Hinsichtlich des achten Satzes der zitierten Schrift Kants, worin dieser Gedanken zur Vervollkommnung einer »Staatsverfassung zum Zweck eines weltbürgerlichen Zustandes « entfaltet, ${ }^{20}$ stützt sich Erzberger auf das Ende des Absatzes, in dem der Königsberger Philosoph zu bedenken gibt, dass Kriege »mit der Zeit ... zu keiner Entscheidung « mehr führten sowie unheilvolle » Wirkungen auf Wirtschaft und Finanzen « hätten, so dass »notwendigerweise die Organisation eines großen Staatskörpers « erfolge, wovon es in der »Vorwelt« kein Beispiel gebe. ${ }^{21}$

Seine rüstungskritische Argumentation setzt Erzberger unter Bezugnahme auf Kants 1786 publizierten, etwa 17 Seiten umfassenden Aufsatz Muthmaßlicher Anfang der Menschengeschichte fort, der im Sinne einer anthropologischen Zusatzreflexion als geschichtsphilosophische Ergänzungsschrift hinsichtlich der zwei Jahre zuvor veröffentlichten Idee zu einer allgemeinen Geschichte ... gesehen werden kann. ${ }^{22}$ Ohne auf die zentralen Überlegungen Kants in dessen Schrift Muthmaßlicher Anfang der Menschengeschichte einzugehen, bei der es im Kern um »den Übergang vom natürlichen zum vernünftigen Menschen « geht $t^{23}$, verweist Erzberger darauf, wie Kant hier »von dem Druck « spreche, »den die unaufhörlich wachsenden Rüstungen auf die Völker « ausübten ${ }^{24}$ und zitiert wörtlich eine Passage aus der »Schluss-Anmerkung « in Kants Aufsatz. Darin werden ständige militärische Rüstungen als »die größten Uebel« gebrandmarkt, »welche gesittete Völker« drückten, und es wird kritisiert, dass somit »Kräfte des Staates«, die zur Förderung der Kultur gebraucht würden, nicht mehr zur Verfügung stünden. Somit werde auch »der Freiheit ... Abbruch getan « und die Vorsorgepflicht des Staates unter Verweis auf äußere Gefahr »in eine unerbittliche Härte der Forderungen verwandelt «.25

18 Immanuel Kant, Idee zu einer allgemeinen Geschichte in weltbürgerlicher Absicht, nach: Erzberger, Der Völkerbund. Der Weg zum Weltfrieden, aaO. (FN 2), S. 66 (Hervorhebung bei Erzberger).

19 Vgl. ebd., S. 67.

20 Vgl. Drube, "Idee zu einer allgemeinen Geschichte in weltbürgerlicher Absicht. Eine geschichtsphilosophische Abhandlung zwischen Zeitkritik und Zukunftsvision« in: Stammen, aaO. (FN 15), S. 79.

21 Vgl. Erzberger, Der Völkerbund. Der Weg zum Weltfrieden, aaO. (FN 2), S. 67.

22 Vgl. Andreas Frei, »Religion und Vernunft. Kants Interpretation der Genesis in Muthmaßlicher Anfang der Menschengeschichte « in: Stammen, aaO. (FN 15), S. 87.

23 Vgl. ebd., S. 97.

24 Vgl. Erzberger, Der Völkerbund. Der Weg zum Weltfrieden, aaO. (FN 2), S. 67 (Hervorhebung bei Erzberger).

25 Immanuel Kant, Muthmaßlicher Anfang der Menschengeschichte, nach: Erzberger, Der Völkerbund. Der Weg zum Weltfrieden, aaO. (FN 2), S. 67f. (Hervorhebung bei Erzberger). 


\section{d) Relevanz völkerrechtlicher Überlegungen Kants für Erzbergers Friedensdenken}

Als »außerordentlich wichtig « bezeichnet Erzberger Kants Aussagen über »die Form des Völkerbundes«, wie sie sich in dessen Abhandlung Über den Gemeinspruch: Das mag in der Theorie richtig sein, tangt aber nicht für die Praxis finden. Dieser Schrift, in der Kant die »im Titel genannte Redensart ... zum Anlass « nimmt, »um in nuce seine praktische Philosophie darzustellen $\ll^{26}$, widmet Erzberger vergleichsweise viel Raum, und er zitiert davon Stellen aus Teil III, in der das »Verhältnis der Theorie zur Praxis im Völkerrecht ... « erörtert wird. ${ }^{27}$ Kant warnt darin vor »dem sschrecklichen Despotismus< einer weltbürgerlichen Verfassung unter einem Oberhaupt«, was Erzberger mit Blick auf die von ihm befürchtete Entwicklung im Sinne »eines Völkerbundes unter Führung einer Mächtegruppe«, vermutlich der Entente, aktualisierend interpretiert. Dagegen trete der Philosoph für eine Staatenföderation "nach einem gemeinschaftlich verabredeten Völkerrecht" ein..$^{28} \mathrm{Um}$ aus der Not beständiger Kriege herauszukommen, die sich zu vervielfältigen drohten, steigende Kosten verursachten und die Ersparnisse des Friedens aufzehrten, müssten die Staaten nach Kant »selbst wider Willen « in »eine weltbürgerliche Verfassung « treten. ${ }^{29}$ Es sei nötig, jeden Staat »in seinem Innern « so zu organisieren, dass »das Volk ... die entscheidende Stimme habe, ob Krieg sein solle oder nicht «.30 Außerdem hebt Erzberger Kants Polemik "gegen das Prinzip des Gleichgewichts der Mächte« hervor. Die von ihm benutzte Metapher eines allzu künstlich austarierten Hauses, das einstürzte »als sich ein Sperling darauf setzte«, sei eine treffende Charakterisierung der kriegsauslösenden Bündniskonstellation um 1914. ${ }^{31}$ Dass der Philosoph in seinem Essay Über den Gemeinspruch begrifflich vorübergehend den »Völkerstaat « gegenüber dem »Völkerbund « bevorzugt ${ }^{32}$ bleibt bei dem Zentrums-

26 Karl-Heinz Nusser, "Immanuel Kant. Über den Gemeinspruch: Das mag in der Theorie richtig sein, tangt aber nicht für die Praxis « in: Theo Stammen / Gisela Riescher / Wilhelm Hofmann (Hg.), Hauptwerke der politischen Theorie, Stuttgart 1997, S. 248.

27 Die genaue Überschrift von Teil III der Kant-Abhandlung lautet: »Vom Verhältnis der Theorie zur Praxis im Völkerrecht, in allgemein-philanthropischer, d.i. kosmopolitischer Absicht betrachtet " nach: Julius Ebbinghaus (Hg.), Immanuel Kant. Über den Gemeinspruch: Das mag in der Theorie richtig sein, tangt aber nicht für die Praxis, Frankfurt am Main 1992, S. 60.

28 Vgl. Erzberger, Der Völkerbund. Der Weg zum Weltfrieden, aaO. (FN 2), S. 68 (Hervorhebung bei Erzberger - im angegebenen Kant-Text ist davon lediglich Völkerrecht hervorgehoben).

29 Vgl. Erzberger, Der Völkerbund. Der Weg zum Weltfrieden, aaO. (FN 2), S. 68f. (Hervorhebung bei Erzberger - im angegebenen Kant-Text ist davon lediglich weltbürgerliche hervorgehoben).

30 Kant, Über den Gemeinspruch..., nach: Erzberger, Der Völkerbund. Der Weg zum Weltfrieden, aaO. (FN 2), S. 69 (Hervorhebung bei Erzberger).

31 Vgl. Erzberger, Der Völkerbund. Der Weg zum Weltfrieden, aaO. (FN 2), S. 69 (Hervorhebung bei Erzberger).

32 Vgl. Massimo Mori, »Friede und Föderalismus bei Kant « in: Zeitschrift für Politik 53, Nr. 4 (2006), S. 382: »Kants Lösung des angesprochenen Problems durchläuft eine parabelartige Entwicklung. Obwohl er grundsätzlich stets dem Völkerbund den Vorzug gab, gewann bis Anfang der 1790er Jahre die These des Völkerstaates an Gewicht; danach sprach er sich hingegen immer nachdrücklicher zugunsten des Völkerbundes aus.« 
politiker ausgeblendet, zumal sich Kant in seiner nun zu thematisierenden berühmten Friedensschrift wieder eindeutig für den letzteren Begriff entscheidet.

\section{e) Über Erzbergers Rezeption von Kants Schrift Zum ewigen Frieden}

Hinsichtlich des für die Friedensproblematik allgemein als zentral geltenden Kant-Textes Zum ewigen Frieden. Ein philosophischer Entwurf ${ }^{33}$ aus dem Jahr 1795 erläutert Erzberger dessen Grundintention, Grobstruktur und Kernaussagen. Es gehe dem Königsberger von der Zielsetzung her um eine »internationale Organisation der Nationen ${ }^{3{ }^{3}}$ Erzberger gibt eine Kurzerläuterung der Schrift gemäß der zwei Hauptabschnitte: sechs Präliminarartikel im ersten sowie drei Definitivartikel im zweiten Teil, ohne allerdings Präambel, die beiden Zusätze und den Anhang zu erwähnen. Worin der Zentrumspolitiker in den Artikeln der beiden Hauptteile die Schwerpunkte sieht, lässt sich an seiner Inhaltskizzierung nachvollziehen:

Der erste Präliminarartikel fordere den Verzicht auf einen »Friedensschluss ... der mit dem geheimen Vorbehalt des Stoffs zu einem künftigen Kriege gemacht worden sei«, womit Kant »jeden Gewaltfrieden « verpöne, »der zu einem Revanchekrieg Anlaß geben « könne. ${ }^{35}$ Im zweiten Präliminarartikel wende er "sich ... gegen die Behandlung eines Staates lediglich als Objekt der Staatskunst « und fordere »die Uebereinstimmung von Regierung und Volk «. ${ }^{36}$ Der dritte Präliminarartikel beinhalte Kants Forderung nach dem »Aufhören der stehenden Heere «, ${ }^{37}$ wozu Erzberger eine längere Passage zitiert. Darin ist u. a. davon die Rede, dass stehende Heere »andere Staaten unaufhörlich mit Krieg durch die Bereitschaft« bedrohten, »immer dazu gerüstet zu erscheinen «. ${ }^{38}$ Solche Heere reizten zum Wettrüsten und würden »selbst $U r$ sache von Angriffskriegen «. »Ganz anders « sei es mit der freiwillig »periodisch vorgenommenen Uebung der Staatsbürger in Waffengewand «, ${ }^{39}$ also einem Milizsystem ${ }^{40}$, um das »Vaterland ... gegen Angriffe von außen zu sichern. $\aleph^{41}$ Gegen rüstungsbedingte »Staatsschulden«bzw. die »Aufnahme von Krediten zur Kriegführung « wende

33 Bezüglich Kants Friedenstheorie vertritt Nusser allerdings den Standpunkt, diese sei »nicht so sehr in seiner Schrift >Zum ewigen Frieden entwickelt, als in der Rechtslehre der Metaphysik der Sitten", während sich erstere »vor allem auf die Auslegung der Naturabsicht « stütze; vgl. Karl-Heinz Nusser, »Kant, Rawls und die >Revolutionen des Friedens<. Kants Nähe zur realistischen Interpretation der internationalen Beziehungen« in: Zeitschrift für Politik 44, Nr. 3 (1997), S. 366.

34 Erzberger, Der Völkerbund. Der Weg zum Weltfrieden, aaO. (FN 2), S. 69 (Hervorhebung im Original).

35 Vgl. ebd. S. $69 f$ (Hervorhebung bei Erzberger).

36 Ebd., S. 70 (Hervorhebung bei Erzberger).

37 Ebd. (Hervorhebung bei Erzberger).

38 Immanuel Kant, Zum ewigen Frieden..., nach: Erzberger, Der Völkerbund. Der Weg zum Weltfrieden, aaO. (FN 2), S 70 (Hervorhebung bei Erzberger).

39 Ebd. (Hervorhebung bei Erzberger).

40 Vgl. Andreas Kleiner, "Norm und Wirklichkeit in Zum ewigen Frieden « in: Stammen, aaO. (FN 15), S. 152.

41 Kant, Zum ewigen Frieden..., nach: Erzberger Der Völkerbund. Der Weg zum Weltfrieden, aaO. (FN 2), S. 70. 
sich Kants vierter Präliminarartikel; im fünften geißle er »die Interventionspolitik« und im sechsten dieser Artikel fordere der Philosoph, auf »solche Feindseligkeiten « zu verzichten, »welche das wechselseitige Zutrauen im künftigen Frieden unmöglich « machten; damit werde »also ... für eine gewisse Beschränkung in den Kriegsmitteln « plädiert, wie »sie ... durch völkerrechtliche Abmachungen geregelt « sei. ${ }^{42}$

$\mathrm{Zu}$ Erzbergers Rezeption von Kants Präliminarartikeln, mit denen dieser gewissermaßen die »negativen Bedingungen des Friedens « ${ }^{43}$ umreißt, kann festgestellt werden, dass der Zentrumspolitiker der dritten dieser Vorbedingungen, also der Abrüstung bzw. Rüstungsbegrenzung, den meisten Platz eingeräumt hat. Dies entspricht der Tatsache, dass auch in Erzbergers Völkerbundkonzept dieses Element nach dem des Obligatorischen Schiedsgerichts einen hervorgehobenen Stellenwert einnimmt. Kants interessante Unterscheidung zwischen sofort geltenden (unbedingten) und allmählich umzusetzenden Forderungen der Präliminarartikel bleibt in Erzbergers Darstellung unberücksichtigt.

Die im zweiten Hauptabschnitt von Kants Friedensschrift enthaltenen drei Definitivartikel, welche »im Gegensatz zu den Präliminarartikeln Normen eines längerfristigen Prozesses « ${ }^{44}$ darstellen, finden sich bei Erzberger im Wesentlichen wie folgt umrissen: Im ersten dieser Artikel erhebe der Königsberger Denker die Forderung nach einer republikanischen Verfassung in jedem Staate, womit ein Rechtsstaat gemeint sei, bei dem die Bürger einen »verfassungsmäßigen Anteil ... an der Staatsgewalt« hätten. Das Völkerrecht habe Kant zufolge »auf einen Föderalismus freier Staaten gegründet " zu sein, wie in seinem zweiten Definitivartikel verlangt werde..$^{45}$ Daraus, dem »Herzstück der gesamten Friedensschrift «, ${ }^{46}$ zitiert Erzberger jene Passage, in der Kant ausdrücklich das Anliegen der »Erbaltung und Sicherung der Freiheit eines Staates für sich selbst und zugleich anderer « ${ }^{47}$ verbündeter Staaten im angestrebten Völkerbund betont. Durch den dritten Definitivartikel mit dem Postulat eines Weltbürgerrechts und der Beschränkung auf allgemeine »Hospitalität« wolle Kant »jedermann Sicherheit gewähren, in welchem Lande er sich auch« befinde, zugleich »die außereuropäischen Länder vor dem Ausbeutungs- und Kolonialsystem einzelner (europäischer) Mächte ... schützen.«48 Allerdings nutzt Erzberger

42 Vgl. Erzberger, Der Völkerbund. Der Weg zum Weltfrieden, aaO. (FN 2), S. $70 f$ (Hervorhebung bei Erzberger).

43 Kleiner, »Norm und Wirklichkeit in Zum ewigen Frieden « in: Stammen, aaO. (FN 15), S. 151.

44 Ebd., S. 156.

45 Vgl. Erzberger, Der Völkerbund. Der Weg zum Weltfrieden, aaO. (FN 2), S. 71 (Hervorhebung bei Erzberger).

46 Kleiner, »Norm und Wirklichkeit in Zum ewigen Frieden « in: Stammen, aaO. (FN 15), S. 158.

47 Kant, Zum ewigen Frieden..., nach: Erzberger, Der Völkerbund. Der Weg zum Weltfrieden, aaO. (FN 2), S. 71 (Hervorhebung bei Erzberger).

48 Erzberger, Der Völkerbund. Der Weg zum Weltfrieden, aaO. (FN 2), S. 71. Eine Modifikation der strikten Verurteilung des Kolonialismus habe Kant später vorgenommen meint Heinz Kluss, »Immanuel Kant und die Reichweite der Kanonen. Die Abkehr von der Illusion des ewigen Friedens « in: Internationale Politik, Nr. 11-12 (2004), S. 155-162. 
das hier aufgezeigte Potenzial nicht zur Formulierung einer konsequent kritischen Position gegenüber dem Kolonialismus, sondern bleibt zeitbefangen - trotz früher Kritik an seinen Auswüchsen und Widersprüchen ${ }^{49}$ - dessen prinzipieller Befürworter, was sich auch in seinem Völkerbundkonzept zeigt. ${ }^{50}$

Insgesamt ist hinsichtlich Erzbergers Rezeption von Kants Friedensschrift festzustellen, dass er deren die Abrüstung betonenden Präliminarartikel und den zweiten Definitivartikel als zentrales Begründungselement des Völkerbundgedankens durch ein die »Idee der Föderalität « ${ }^{51}$ betonendes Zitat besonders hervorhebt.

\section{f) Rechtsentwicklung als Mittel der Versittlichung zur Überwindung des Krieges}

Mit Blick auf Kants Rechtslehre, speziell den Völkerrechtsteil seiner Metaphysik der Sitten - auch als ein »Epilog « zur Friedensschrift eingeordnet ${ }^{52}$ - verweist Erzberger darauf, dass der Philosoph »die allmähliche Annäherung an die Herbeiführung des ... ewigen Friedens als das letzte Ziel des ganzen Völkerrechts « erkläre. ${ }^{53}$ Der berühmte Denker spreche »von einem Verein einiger Staaten« zur Erhaltung des Friedens, den man gemäß eines von Erzberger präsentierten Kant-Zitates »den permanenten Staatenkongress nennen « dürfe und $\mathrm{zu}$ dem sich weitere Staaten gesellen könnten. ${ }^{54}$ Dabei handle es sich um eine »zu aller Zeit auflösliche ${ }^{55}$ Zusammentretung « ohne feste Staatsverfassung, also um einen lockeren Zusammenschluss, »durch welchen allein die Idee eines zu errichtenden öffentlichen Rechts der Völker, ibre Streitigkeiten auf zivile Art, gleichsam durch einen Prozeß, nicht auf barbarische (nach Art der Wilden), nämlich durch den Krieg zu entscheiden, realisiert werden kann. ${ }^{56}$

Das Bemühen, dem Krieg - der von Kant als größtes "Hindernis des Morali-

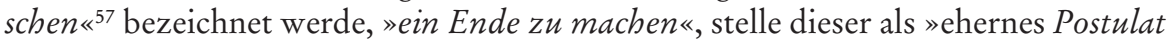
des Sittengesetzes « dar, selbst »wenn theoretisch der ewige Friede nicht erreichbar»

49 Vgl. Christian Leitzbach, Matthias Erzberger. Ein kritischer Beobachter des Wilhelminischen Reiches 1895-1914, Frankfurt am Main/Berlin/Bern/New York/Paris/Wien 1998, S. 293-398.

50 Vgl. Erzberger, Der Völkerbund. Der Weg zum Weltfrieden, aaO. (FN 2), insbesondere Kapitel XI. Kolonialfragen, S. 145-151.

51 Ebd., S. 171 (Hervorhebung bei Erzberger, Föderalität hebt auch Kant hervor).

52 Zahn vertritt die Ansicht, dass Kants »Abschnitte der spätesten Schriften wie die >Anthropologie in pragmatischer Absicht< oder auch der >Metaphysik der Sitten $<. .$. wie ein die Friedensschrift vom moralischen Gesichtspunkt einschränkender Epilog « gelesen werden könnten; vgl. Zahn, »Kant und die Problematik des neuesten Friedensbegriffs « in: Autorenteam ... (Hg.), Frieden im Spektrum ... aaO. (FN 17), S. 31.

53 Vgl. Erzberger, Der Völkerbund. Der Weg zum Weltfrieden, aaO. (FN 2), S. 71.

54 Vgl. Erzberger, Der Völkerbund. Der Weg zum Weltfrieden, aaO. (FN 2), S. 72 (Hervorhebung im Original).

55 Unterschiedlich, auch als »ablösliche«, wiedergegeben.

56 Immanuel Kant, Die Metaphysik der Sitten (Rechtslehre), zitiert nach: Erzberger, Der Völkerbund. Der Weg zum Weltfrieden, aaO. (FN 2), S. 72 (Hervorhebung bei Erzberger).

57 Immanuel Kant, Der Streit der Fakultäten, nach: Erzberger, Der Völkerbund. Der Weg zum Weltfrieden, aaO. (FN 2), S. 72 (Hervorhebung bei Erzberger). 
sei. ${ }^{58}$ In einem weiteren von Erzberger angeführten Zitat aus dem Schlussteil (»Beschluss «) von Kants Metaphysik der Sitten wird die "allgemeine und fortdauernde Friedensstiftung « als der ganze »Endzweck der Rechtslehre innerhalb der Grenzen der bloßen Vernunft « bezeichnet. ${ }^{59}$ Der anvisierte Friedenszustand sei nach Kant "allein der unter Gesetzen gesicherte Zustand des Mein und Dein in einer Menge benachbarter Menschen « - anzustreben sei er »nicht revolutionsmäßig, durch einen Sprung ... durch gewaltsame Umstürzung ..., sondern durch allmähliche Reform nach festen Grundsätzen « ${ }^{60}$ Erzberger resümiert, Kant komme »auf Grund seiner Vernunfterkenntnisse zu derselben Bewertung des Krieges und derselben Forderung nach Einrichtungen « zu dessen Einschränkung, ja Beseitigung, »wie die Päpste«, und er nennt zusätzliche Vertreter deutscher Geistesgeschichte, wie Schlegel, Schelling, Herder, Novalis und Fichte, die in eine ähnliche Richtung wie Kant gedacht hätten. ${ }^{61}$ Statt hier jedoch möglichen Parallelen zu den von Erzberger genannten Denkern aus dem 19. Jahrhundert nachzugehen, sei nun der Blick ins 20. und über die Schwelle zum 21. Jahrhundert gelenkt, um die Frage zu erörtern, inwiefern bei einem bedeutenden Zeitgenossen der Gegenwart, dem Friedensforscher Dieter Senghaas, Bezüge auf Immanuel Kant erkennbar bzw. wirksam werden.

\section{Senghaas und Immanuel Kant}

\section{a) Vom ewigen Frieden bei Kant Zum irdischen Frieden bei Senghaas}

Als Dieter Senghaas 1995, zweihundert Jahre nach dem Erscheinen des berühmten philosophischen Entwurfs Zum ewigen Frieden, mit dem Band Den Frieden denken eine Sammlung grundlegender Texte ganz unterschiedlicher Autoren zu dieser Problematik herausgab, erinnerte er mit der vorangestellten Widmung Kant zu ehren ausdrücklich an den großen deutschen Aufklärer. Warum dieser für Senghaas »ein beispielhaft konsequenter Friedensdenker « gewesen ist, begründete er so:

Für Kant »war selbstverständlich, was allgemein selbstverständlich sein sollte: Das Maß des Friedens ist der Frieden selbst. « ${ }^{62}$

Im Kern ist damit gemeint, dass es eines positiven Entwurfes bedarf, um Frieden als dauerhafte Ordnung vorzubereiten, also eines Konzeptes, das sich zielstrebig daran ausrichtet, wodurch und wie sich Frieden konstituiert, ${ }^{63}$ anstatt sich lediglich auf

58 Vgl. Erzberger, Der Völkerbund. Der Weg zum Weltfrieden, aaO. (FN 2), S. 72 (Hervorhebung im Original).

59 Vgl. ebd. (Hervorhebung bei Erzberger).

60 Kant, Die Metaphysik der Sitten (Rechtslehre), nach: Erzberger, Der Völkerbund. Der Weg zum Weltfrieden, aaO. (FN 2), S. 72 (Hervorhebung bei Kant).

61 Vgl. Erzberger, Der Völkerbund. Der Weg zum Weltfrieden, aaO. (FN 2), S. $72 \mathrm{f}$.

62 Dieter Senghaas (Hg.), Den Frieden denken. Si vis pacem, para pacem, Frankfurt am Main 1995, Vorwort, S. 16 (Hervorhebung im Original).

63 Die betreffende, von Senghaas formulierte friedenstheoretische Grundfrage hat sich auch in einem Buchtitel niedergeschlagen: Jörg Calließ (Hg.), Wodurch und wie konstitwiert sich Frieden? Das zivilisatorische Hexagon auf dem Prüfstand, Loccum 1997 (Dokumentation einer Tagung der Evangelischen Akademie Loccum vom 13.-15. November 1996). 
Versuche der Unterdrückung von Kriegen mit primär militärischen Mitteln - v. a. der Bereitstellung abschreckender Rüstung gegen potenzielle Feinde - zu beschränken.

In Senghaas' Buch Zum irdischen Frieden zeigt sich die Bezugnahme auf den Königsberger Philosophen äußerlich schon mit der Anspielung auf den Titel von dessen Schrift Zum ewigen Frieden. Aber auch die von Senghaas in Analogie zu Kant gewählte Parallelstruktur mit einer Gliederung der Publikation gemäß damaliger völkerrechtlicher Verträge ${ }^{64}$ in Präliminar- und Definitivartikel sowie mit Zusätzen und einem Anhang kann als Gleichnis dafür verstanden werden, dass es Senghaas als anregend betrachtet, symbolisch an Kants klassischen Entwurf anzuknüpfen, aber auch für notwendig, die Herausforderung des Friedens den veränderten Realitäten entsprechend in wesentlichen Aspekten neu zu bedenken und konzeptionell zu aktualisieren.

So kann sich nach Senghaas ein heute »zeitgemäßer Traktat zum irdischen Frieden in der Analyse nicht nur auf das sich aus der so genannten >Anarchie der Staatenwelt ergebende Sicherheitsdilemma « beschränken, sondern muss vor dem Hintergrund eines inzwischen global »zum Problem gewordenen Entwicklungsdilemmas « im Wesentlichen von einer Vierteilung der Welt ausgehen. ${ }^{65}$ Deren tendenziell unterscheidbare Zonen müssten hinsichtlich der Friedensproblematik einerseits differenziert und »kontextsensibel « analysiert, andererseits aber auch in der Zusammenschau ihrer Gesamtstruktur erfasst und bearbeitet werden. Problemadäquate Friedensprogrammatiken lassen sich demnach »nicht durch eine Reduktion von Komplexität« gewinnen, sondern setzen vielmehr »deren Steigerung « voraus. ${ }^{66}$

\section{b) Kritik von Schein-Rationalität als Prämisse zur Ermöglichung friedenspolitischer Vernunft}

Ähnlich wie die berühmten Kritiken Kants für dessen Gesamtwerk kennzeichnend sind, kommt Senghaas' Kritik an Kolonialismus/Imperialismus, ${ }^{67}$ Abschreckungspolitik, ${ }^{68}$ Rüstung und Militarismus ${ }^{69}$ für sein gesamtes Friedensdenken eine grundlegende Bedeutung zu. In gewisser Analogie mit der zu emanzipatorischen Impulsen anregenden Kant-Devise, sich des eigenen Verstandes zu bedienen, inspi-

64 Vgl. Hans-Otto Mühleisen, »Zum ewigen Frieden. Ein philosophischer Entwurf in: Stammen / Riescher / Hofmann, aaO. (FN 26), S. 251-255.

65 Folgendermaßen: 1) Die OECD-Welt (Welt I), Die neue Zweite Welt (Welt II) = EUErweiterungsländer, ostasiatische und z. T. südostasiatische Gesellschaften, 3) Die Dritte Welt (Welt III), Die Vierte Welt (Welt IV), Länder »mit einem regelrechten Zusammenbruch der öffentlichen Ordnung «, insbesondere in weiten Teilen Schwarzafrikas, nach: Dieter Senghaas, »Die Konstitution der Welt - eine Analyse in friedenspolitischer Absicht « in Leviathan. Zeitschrift für Sozialwissenschaft 31, Nr. 1 (2003), S. 117-152.

66 Vgl. Senghaas, Zum irdischen Frieden..., aaO. (FN 4), S. 7-14.

67 Vgl. v.a.: Dieter Senghaas (Hg.), Imperialismus und strukturelle Gewalt. Analysen über abhängige Reproduktion, Frankfurt am Main 1972.

68 Vgl. v.a.: Dieter Senghaas, Abschreckung und Frieden. Studien zur Kritik organisierter Friedlosigkeit, Frankfurt am Main 1969.

69 Vgl. v a.: Dieter Senghaas, Rüstung und Militarismus, Frankfurt am Main 1972. 
riert Senghaas vor dem Hintergrund des Ost-West-Konfliktes dazu, »den jedem Rüstungswettlauf zugrundeliegenden Verblendungszusammenhang aufzubrechen « und die »Irrationalität von Abschreckungspolitik « zu erkennen, die lediglich »mit dem Schein von Rationalität verkleidet « sei, ${ }^{70}$ denn - so seine Diagnose:

»Drohpolitik produziert Aggressivität, und Aggressivität läßt ihrerseits Drohpolitik wieder als rationale Reaktion erscheinen. «11

Demnach gehört es wesentlich zu den Aufgaben kritischer Vernunft, die Diskrepanz zwischen scheinbarer und wirklicher Rationalität bewusst zu machen, um die sonst drohenden pathologischen Lernprozesse mit ihren gewaltproduzierenden Folgen zu korrigieren und in friedenszuträgliche Bahnen umzulenken. Um die Bedingungen $\mathrm{zu}$ verdeutlichen, unter denen sich »friedenspolitische Vernunft« aktualisieren kann, unternimmt Senghaas einen begrenzten »Vergleich bestimmter struktureller Besonderheiten des Freudschen Modells der individuellen menschlichen Psyche mit bestimmten strukturellen Besonderheiten der ... Staaten, der Interessengruppen innerhalb einer Nation sowie des internationalen Systems « ${ }^{72}$. Zusammenfassend aus seinen diesbezüglichen Überlegungen rät der Friedensforscher »davon auszugehen, dass unter den Bedingungen des späten 20. und des beginnenden 21. Jahrhunderts die >Geistesverfassung ‘ der Staaten prekärer ... als die von Individuen « sei, so »daß die Konstitutionsbedingungen friedenspolitischer Vernunft in der Zukunft nicht einfacher gelagert sein « würden als bisher. ${ }^{73}$ Dennoch nimmt Senghaas mit dem Ende des Ost-West-Konflikts neue Möglichkeiten der Friedensgestaltung wahr, bleibt aber gegenüber klassischen Gleichgewichtsbestrebungen, denen oft friedenssichernde Wirkung zugeschrieben wird - ähnlich wie Kant und Erzberger - in einer kritischen Haltung:

»Aus friedenstheoretischer Perspektive ist bei der Bewältigung des internationalen Sicherheitsdilemmas eindeutig einem institutionalisierten Netzwerk von Kooperation der Vorzug vor den konventionellen Vorkehrungen des zwischenstaatlichen Machtmanagements zu geben, wie sie in Arrangements des Mächtegleichgewichts, der Hegemonialstruktur, der Allianzen und Gegenallianzen usf. zum Ausdruck kommen. Diese letzteren Vorkehrungen taugen nämlich nicht dazu, das Sicherheitsdilemma dauerhaft überwinden zu helfen; im Gegenteil sind sie oft genug für die Verlängerung und Verschärfung des Sicherheitsdilemmas verantwortlich. ${ }^{74}$

70 Vgl. Dieter Senghaas (Hg.), Zur Pathologie des Rüstungswettlaufs. Beiträge zur Friedens- und Konfliktforschung, Freiburg im Breisgau 1970, Vorwort, S. 16.

71 Ebd., S. 36.

72 Karl W. Deutsch / Dieter Senghaas, "Die brüchige Vernunft von Staaten« in: Dieter Senghaas (Hg.), Kritische Friedensforschung, S. 105-158 (2. Auflage), Frankfurt am Main 1972, S. 110. Die überarbeitete, aktualisierte und gekürzte Version des bereits 1971 in Kritische Friedensforschung publizierten Beitrags bildet bei Dieter Senghaas, Zum irdischen Frieden..., aaO (FN 4), Kapitel I, 3. Dritter Abschnitt, welcher friedenspolitische Vernunft ermöglichende Bedingungen erläutert: Wodurch und wie konstituiert sich die Vernunft zum Frieden heute?, S. 81-123 (Hervorhebung im Original).

73 Vgl. ebd., S. 123.

74 Dieter Senghaas, »Die Neugestaltung Europas « in: Aus Politik und Zeitgeschichte, B 18/ 91 (1991), S. 15 (Hervorhebung im Original). 
Damit wird deutlich, wie die Entwicklung positiver friedenspolitischer Perspektiven bei Senghaas eine Kritik der von ihm negativ eingeschätzten Bedingungen voraussetzt.

\section{c) Stiftung positiven Friedens, Rechtsstaatlichkeit und Wege zu Vergemeinschaftung}

Ähnlich wie Kant in seinen Präliminarartikeln Zum ewigen Frieden vor negativen Implikationen warnt, die einen solchen vereiteln und deshalb in den Definitivartikeln der Schrift positive Bedingungen umreißt (republikanische Verfassung bzw. Rechtsstaatlichkeit, einen Föderalismus freier Staaten und ein Weltbürgerrecht), so zeigt Senghaas unter Auswertung historischer Erfahrungen nach der umfassenden Kritik von Störfaktoren des Friedens gangbare Wege in eine gegenwartsorientierte bzw. zukunftsweisende und tendenziell nachhaltige Weltfriedensordnung. In seiner Begrifflichkeit kommt dies dem Übergang von der bloßen Negation des "para bellum«-Denkens zur ausdrücklichen Hinwendung auf eine Position der »para pacem «-Perspektive gleich. Sie konzeptualisiert sich bei dem Friedensforscher theoretisch insbesondere in einer Zusammenstellung von vier sogenannten Komplexprogrammen ${ }^{75}$. Deren erstes benennt »Elemente einer Friedensstruktur «, das zweite kennzeichnet »Frieden als kollektive(n) Lernprozeß der Gemeinschaftsbildung ", das dritte Komplexprogramm - als Zivilisatorisches Hexagon stärker als die übrigen drei in die allgemeine Diskussion gelangt - zeigt sechs miteinander verbundene Bedingungen für die »Zivilisierung der Konfliktbearbeitung « auf ${ }^{76}$ und Komplexprogramm IV verweist auf eine »synthetisierende Leitperspektive mit praxeologischen Implikationen« für globale Friedensgestaltung.

Gemeinsam sind dem Kant'schen wie dem Senghaas'schen Gesamtkonzept insbesondere der zentrale Stellenwert von Rechtsstaatlichkeit sowie die entscheidende Erkenntnis, dass Friede nicht von selbst schon mit der Eliminierung friedensabträglicher Störfaktoren oder Negativ-Bedingungen entsteht, sondern vielmehr der absichtsvollen, zielstrebig-positiven Stiftung bedarf. ${ }^{77}$ Im folgenden Zitat aus einem Beitrag des Sozialwissenschaftlers mit dem bezeichnenden Titel In den Frieden zieben wird dieser entscheidende Zusammenhang hervorgehoben:

»Rechtsstaatlich verfaßte Einzelstaaten finden sich nicht von allein zu einem wie Kant formuliert hätte - >Friedensbund zusammen. Eine Friedensordnung zwischen >Republiken muß wie Kant zu Recht betonte, ausdrücklich gestiftet werden. $\ll^{78}$

75 Vgl. Senghaas, Zum irdischen Frieden ..., aaO. (FN 4), S. 143-161.

76 Im nächsten Unterpunkt näher ausgeführt.

77 Vgl. Kants Vorbemerkungen zum ersten Definitivartikel; in: Kant, Zum ewigen Frieden..., aaO. (FN 5), S. 10. Stiftung von Frieden als politischer »Gründungsakt « ist grundlegend dargelegt bei: Volker Gerhardt, Immanuel Kants Entwurf ,'Zum ewigen Frieden. Eine Theorie der Politik, Darmstadt 1995, S- 74-78.

78 Dieter Senghaas, »In den Frieden ziehen. Neue Aufgaben der Konfliktforschung nach dem Ende des weltbedrohenden Gegensatzes zwischen Ost und West « in: Frankfurter Allgemeine Zeitung (Wochenendbeilage >Bilder und Zeiten<), Nr. 131, 6. Juni 1992. 
Die schon bei Kant angelegte Forderung nach Rechtsstaatlichkeit bildet auch das Fundament für Senghaas' europäischen Friedensplan von 1989/90,79 stellt einen der vier Bausteine im zwei Jahre später präzisierten Friedensprojekt für den Kontinent dar ${ }^{80}$ und bildet schließlich eine von sechs konstituierenden Determinanten für das zivilisatorische Hexagon, ${ }^{81}$ weil Rechtsstaatlichkeit insbesondere das sonst zur Auswucherung neigende Gewaltmonopol des Staates einzuhegen vermag.

Während Kant in seiner Schrift Zum ewigen Frieden die Föderation republikanisch verfasster Staaten in einem Friedensbund anregt sowie Konturen eines Weltbürgerrechts noch aus prämoderner Sicht umreißt, benennt Senghaas vor dem Hintergrund sich modernisierender und politisierbarer Gesellschaften mit seinen (weiteren) Komplexprogrammen die für dauerhaften Frieden heute erforderlichen Schritte interner wie grenzüberschreitender Gemeinschaftsbildung - Überlegungen, die wohl auch für die Entfaltung einer Global Governance-Architektur nutzbar sind. ${ }^{82}$

\section{d) Friedenspolitische Konsequenzen eines grundlegenden sozialstrukturellen Wandels}

Stark unterschiedlich zeigen sich die Welten von Kant und Senghaas in sozialstruktureller Hinsicht: Während zu Kants Zeiten die Gesellschaften noch überwiegend bäuerlich geprägt waren, hat mit den darauf abgelaufenen Industrialisierungsund Modernisierungsprozessen in weiten Bereichen eine Entbäuerlichung bzw. Verstädterung stattgefunden, mit der vormals weitgehend schreib- und leseunkundige Bevölkerungsmehrheiten alphabetisiert, somit in mehrfacher Hinsicht mobil und politisierbar wurden. Diese veränderten sozialen Prämissen waren der alteuropäischen Realität eines Immanuel Kant praktisch fremd, konnten deshalb auch für sein Friedenskonzept keine Rolle spielen. Als logische Hauptkonsequenz aus diesem unterschiedlichen Gesellschaftshintergrund der Konzepte von Kant und Senghaas folgen die von letzterem vorgenommenen Anpassungen an komplexere Herausforderungen, wie sie sich in Senghaas' vier Komplexprogrammen niederschlagen, deren bekanntestes im zivilisatorischen Hexagon repräsentiert wird. Für diese Modellfigur ${ }^{83}$ sind folgende Bausteine konstitutiv und im Idealfall durch positive Rückkoppelungsprozesse sich wechselseitig stabilisierend:

79 Dieter Senghaas, Europa 2000. Ein Friedensplan, Frankfurt am Main 1990.

80 Dieter Senghaas, Friedensprojekt Europa, Frankfurt am Main 1992.

81 Vgl. Dieter Senghaas, Wohin driftet die Welt? Über die Zukunft friedlicher Koexistenz, Frankfurt am Main 1994, insbesondere S. 20-34.

$82 \mathrm{Zu}$ Global Governance: Dirk Messner / Franz Nuscheler, »Das Konzept Global Governance - Stand und Perspektiven « in: Dieter Senghaas / Michèle Roth (Redaktion), Global Governance für Entwicklung und Frieden. Perspektiven nach einem Jabrzehnt. Sonderband zum 20jäbrigen Bestehen der Stiftung Entwicklung und Frieden, Bonn 2006, S. 18-79.

83 Siehe Abbildung 2 am Ende des vorliegenden Beitrags. 
a) ein übergeordnetes Gewaltmonopol (normalerweise im Nationalstaat),

b) dessen rechtsstaatliche Bändigung bzw. Einhegung,

c) vielfältige Interdependenzen und damit verbundene Affektkontrollen,

d) befriedigende Möglichkeiten politischer Partizipation,

e) Verteilungsgerechtigkeit,

f) eine Kultur konstruktiver Konfliktbearbeitung.

Nachdrücklich betont Senghaas Notwendigkeit sowie Chancen eines positiven Zusammenspiels dieser sechs Grundbedingungen für zivilisierte Konfliktbearbeitung innerhalb sich modernisierender bzw. moderner Gesellschaften. Im folgenden Zitat verdeutlicht er den gravierenden Unterschied zur Welt Kants und charakterisiert zugleich Umstände der mit ihrer Veränderung beobachtbaren Emanzipationsprozesse:

»Alle diese Punkte (des zivilisatorischen Hexagons, A.S.) sind nicht Teil des alteuropäischen Verständnisses vor 200 Jahren, und jeder einzelne musste wider Willen mit Hilfe von Kompromissen errungen werden ${ }^{84}$

Bezüglich des mit Globalisierungsprozessen wahrgenommenen Übergangs von der nationalen zur postnationalen Konstellation, sieht Zürn die Ecken des Hexagons auf verschiedenen Ebenen (teils beim Nationalstaat, teils international) angesiedelt und postuliert dementsprechend die Modellfigur als »Mehrebenenhexagon «, welches dann »den institutionellen Rahmen für eine Weltinnenpolitik abgeben« könne. ${ }^{85}$

\section{e) Reichweiten und Intensitäten grenzüberschreitender Gemeinschaftsbildung}

Wie deutlich geworden ist, kann im friedenspolitischen Grundanliegen grenzüberschreitender Vergemeinschaftung zum Aufbau dauerhaften Friedens eine prinzipielle Gemeinsamkeit der Konzepte von Kant und Senghaas gesehen werden. Ein nennenswerter Unterschied liegt allerdings darin, dass Senghaas - mitinspiriert durch die Integrationserfahrungen im Gebiet der EU seit der zweiten Hälfte des 20. Jahrhunderts - relativ weitgehende Formen bzw. Intensitäten der Integration für erstrebenswert hält, die Kant aus systematischen Gründen wohl nicht akzeptiert hätte, da sie über Konföderation hinaus gehen. ${ }^{86}$ Somit beinhaltet das Friedenskonzept von Senghaas in der Konsequenz eine höhere Bereitschaft zum Souveränitätsverzicht beteiligter Staaten, als sie vor rund 200 Jahren Kant vermutlich für wünschenswert bzw. für zumutbar gehalten hätte.

Was die globale Alternative "pluralistische« oder »amalgamierte Sicherheitsgemeinschaft« betrifft - letztere liefe auf eine Art von Weltstaat hinaus -, so lässt Seng-

84 Dieter Senghaas am 16. 2. 2004 bei einem Vortrag in der Stadthalle Biberach an der Riss, nach: Wolfgang Heinzel, »Senghaas und Kant. Sechs Voraussetzungen zur Konfliktbearbeitung in: Schwäbische Zeitung, 25. Februar 2004 (Ausgabe Biberach).

85 Vgl. Michael Zürn, »Frieden umfassend denken« in: Leviathan. Berliner Zeitschrift für Sozialwissenschaft 33, Nr. 4 (2005), S. 428-438.

86 So Dieter Senghaas in persönlichen Gesprächen mit dem Autor. 
haas ähnlich wie Kant eine Präferenz für die losere Form der »pluralistischen Sicherheitsgemeinschaft « erkennen. Die Verschmelzung in einem Weltstaat ließe nach Kant die Gefahr eines seelenlosen Despotismus (»Despotism«) und letztlich Anarchie befürchten. ${ }^{87}$ Senghaas betont als Grund für seine vergleichbare Position die mannigfaltige Zerklüftung globaler Realität bzw. die gravierenden Defizite an homogenen und kohärenten Handlungszusammenhängen samt geringer Aussichten auf baldige Homogenisierung, ${ }^{88}$ und er macht darauf aufmerksam, dass schon der Philosoph aus Königsberg die sich heute verstärkt stellende Herausforderung grundsätzlich hatte erkennen lassen:

»Untereinander in wirksame Verhältnisse kommen - das war Immanuel Kants begriffliche Umschreibung für jene Erscheinung, die heute landläufig als sich erweiternde und vertiefende weltweite Interdependenzen bezeichnet wird. Und schon Kant hatte eine verhaltene Vorahnung in seinen Schriften über das, was nunmehr ganz offenkundig ist und dennoch im Friedensdiskurs selten oder nur beiläufig registriert wird: die Tatsache, dass suntereinander in wirksame Verhältnisse kommen< zu extrem unterschiedlichen Lagen in der Welt führt und folglich auch eine entsprechend differenzierte Lagebeurteilung samt differenzierter friedenspraktischer Folgerungen erforderlich macht. ${ }^{89}$

Gemäß der von Senghaas getroffenen Differenzierung globaler Realität in vier Teilwelten mit spezifischen Merkmalen ergeben sich verschiedene friedenspolitische Imperative. ${ }^{90} \mathrm{Zu}$ entwickelnde Konzepte hätten v.a. auch Probleme einzubeziehen, wie sie sich aus vermehrt asymmetrischen Konfliktszenarien ergeben. ${ }^{91}$ Statt dies auszuführen, wird im Folgenden eine vergleichende Bilanz über Analogien und Unterschiede zu den skizzierten Kant-Bezügen bei Erzberger und Senghaas gezogen.

\section{3) Unterschiede und Analogien in Kant-Bezügen von Erzberger und Senghaas - Fazit}

Mit den vorangegangenen Ausführungen dürfte deutlich geworden sein, wie bei den Friedenskonzepten von Matthias Erzberger und Dieter Senghaas in je spezifischer Weise Verbindungen zur Aufklärungsphilosophie von Immanuel Kant vorliegen. Während Erzberger zur Begründung von Teilen seines friedenspolitischen Konzeptes (z.B. in Gleichgewichtskritik, Abrüstungs- und Völkerbundprogrammatik) auf entsprechende Stellen der Schriften Kants argumentativ zurückgreift und

87 Vgl. Senghaas, Wobin driftet die Welt?..., aaO. (FN 81), S. $35 f$.

88 Vgl. ebd., S. 36. Eine ähnlich ablehnende Haltung gegenüber Weltstaatsvorstellungen vertrat übrigens auch Erzberger, Der Völkerbund. Der Weg zum Weltfrieden, aaO. (FN 2), S. 162.

89 Senghaas, Zum irdischen Frieden..., aaO. (FN 4), S. 294.

90 Vgl. Senghaas, »Die Konstitution der Welt - eine Analyse in friedenspolitischer Absicht «, aaO. (FN 65), S.148-152.

91 Vgl. Herfried Münkler, »Elemente einer neuen Sicherheitsarchitektur. Die Weltordnungsstrukturen haben sich grundlegend verändert. Eine Neuanpassung der deutschen Sicherheitssysteme ist überfällig « in: Internationale Politik, Nr. 5 (2007), S. 6-14. 
sie bisweilen ausführlich zitiert, sind friedenskonzeptionelle Entsprechungen zwischen Senghaas und Kant auf einer eher abstrakt-grundsätzlichen Ebene auszumachen. Dafür können Senghaas' Beiträge prinzipiell in sich vergleichsweise schlüssig und widerspruchsfrei als konsequente Fortführung des friedenspolitischen Projektes im Sinne Kant'scher Aufklärung verstanden werden. Erzberger hingegen versucht eher bestimmte Elemente daraus in sein Friedenskonzept pragmatisch zu integrieren, das im Wesentlichen auf der Idee eines Völkerbundes beruht. Insgesamt jedoch steht Erzbergers Friedensdenken weniger stringent in der Tradition Kant'scher Aufklärungsphilosophie. Insbesondere dadurch, dass Erzbergers politische Ethik auch mehr oder weniger antiliberale Denkweisen - hergeleitet aus römisch-katholischer Dogmatik - beinhaltet, ${ }^{92}$ scheint sie weniger als das Senghaas'sche Friedenskonzept (als Zivilisierungsprojekt) mit dem Projekt der Aufklärung insgesamt zu harmonieren. Anders als Erzberger, der grundsätzlich Kants Idee zu einem Völkerbund im Sinne des Inbegriffs friedensorientierter Vernunft als »realpolitische Notwendigkeit « konzeptualisiert, reflektiert Senghaas zunächst Konstitutionsbedingungen friedenspolitischer Vernunft, um von dieser kritisch geprüften Basis aus differenzierte und zu einem Gesamtkonzept integrierbare Teilantworten auf die Grundfrage zu entwickeln:

»Wodurch und wie konstituiert sich Frieden unter den komplexen Bedingungen der Gegenwart? «93

Gleichwohl finden sich hinsichtlich friedenstheoretischer Bezüge zu Kant bzw. zu seiner Philosophie bemerkenswerte Parallelen:

\section{a) Der Rekurs auf die klassische Schrift, Zum ewigen Frieden}

Kants Schrift Zum ewigen Frieden ist sowohl bei Erzberger als auch bei Senghaas als wegweisendes Dokument erkennbar, wenngleich es anders rezipiert und friedenskonzeptionell unterschiedlich verarbeitet wird. Erzberger untermauert damit das von ihm propagierte Völkerbundkonzept, Senghaas knüpft zwar mit seinem Buch Zum irdischen Frieden nicht nur semantisch an »das Kantische Projekt« an, sondern artikuliert auch mit dem benutzten Stilmittel der strukturellen Parallelität ${ }^{94}$ angelehnt an Kants Zum ewigen Frieden die Absicht, rund 200 Jahre später mit konsequent theoriegesättigten Beiträgen »eine weltkundige zeitgemäße Konkretion « vorzulegen. ${ }^{95}$ Für beide Autoren gewinnen daraus insbesondere Kants Definitivartikel ein besonderes Gewicht, insofern z. B. Erzberger deren zweiten zum Kern seines

92 Vgl. Klaus Epstein, Matthias Erzberger und das Dilemma der deutschen Demokratie, Berlin/Frankfurt am Main 1962, S. 280.

93 Senghaas, Zum irdischen Frieden..., aaO. (FN 4), S. 24 (Hervorhebung im Original).

94 Mit der Synopse in Abbildung 1 am Ende des vorliegenden Beitrags soll die strukturelle Analogie illustriert, aber auch sichtbar werden, dass es sich inhaltlich lediglich um begrenzte Entsprechungen handelt.

95 Vgl. Senghaas, Zum irdischen Frieden..., aaO. (FN 4), Text auf dem Buchrücken. 
Gesamtkonzeptes macht und Senghaas erklärt, dass sein Buch Zum irdischen Frieden »im Grunde genommen« als »Definitivbestimmung des Friedens « zu verstehen sei. ${ }^{96}$

\section{b) Von "para bellum«-Kritik zu "para pacem«-Perspektiven}

Eine von Senghaas systematisch entfaltete und bei Erzberger ebenfalls deutlich konturierte Kritik von "para bellum «-Fixierungen (z. B. Ablehnung von Gleichgewichtsdogmatik und des damit verbundenen Vertrauens in militärische Rüstung bzw. Abschreckung als angebliche Friedensgaranten) ist schon in der Friedensphilosophie Kants angelegt. So grundlegend aber bei allen drei Autoren eine Kritik des Unfriedens bzw. seiner Ursachen auch ist (»Präliminar«-Überlegungen), so beschränken sie sich doch nicht auf dessen Negation bzw. Bekämpfung, sondern formulieren Positionen zur Entwicklung einer Perspektive dauerhaften Friedens (»Definitiv«-Überlegungen). Die in Kants Denken im Sinne Senghaas'scher Terminologie hineinprojizierbare "para pacem«-Perspektive wird von Kant (in den drei Definitivartikeln) zentral als Völkerbundidee definiert und diese von Erzberger im gegebenen Zeithorizont (z.B. mit kolonialem Ballast) politisch konzeptualisiert. Senghaas hingegen begründet und entfaltet die von ihm so genannte "para pacem «-Perspektive wissenschaftlich sowie neueren Erkenntnissen und der veränderten Weltlage entsprechend differenziert, v.a. unter Berücksichtigung des mit Modernisierungsprozessen verbundenen sozialen Wandels. Während Erzberger insbesondere Kants allgemeine Idee eines Föderalismus »republikanisch « verfasster Staaten zum konkreten Friedenskonzept im Sinne seiner Vorstellungen für eine Weltorganisation auszuarbeiten sucht, bleiben Senghaas' Überlegungen offener und trotz des Plädoyers für eine rechtsstaatsanaloge (Re-) Form der Vereinten Nationen ${ }^{97}$ nicht primär auf eine bestimmte Variante globaler bzw. regionaler Organisation(en) beschränkt; vielmehr betreffen seine Überlegungen stärker generalisierbare Prozesse zu politischer Vergemeinschaftung, mit denen prinzipielle Strukturen dauerhaften Friedens wahrscheinlicher werden. Dem von Kant ausdrücklich betonten Grundgedanken, dass dauerhafter Friede einer systematischen Stiftung und Vorbereitung bedarf, wird von beiden Autoren auf je spezifische Weise entsprochen.

\section{c) Wachsende Friedensrelevanz von Handel, Wirtschaft und Entwicklung}

Die bei Kant formulierte Vorstellung, dass mit dem »Handelsgeist ... Kriege nicht zusammen bestehen « könnten, ${ }^{98}$ findet in Erzbergers Friedenskonzept insofern eine

96 Vgl. Steffen Graefe, Auf den Spuren von Immanuel Kant. Dieter Senghaas: »Zum irdischen Frieden «, (Interview im Deutschlandfunk vom 6.9.2004).

97 Vgl. Dieter Senghaas im Interview mit Volker Dieckmann:»Kampf aller gegen alle< muss beendet werden. Versorgung der Bevölkerung vordringlich - Welches Recht soll welche Ordnung schaffen? « in: Nürnberger Nachrichten, 17. April 2003, auch dokumentiert in: Siegel, Ideen zur Friedensgestaltung..., aaO. (FN 3), S. 490.

98 Vgl. Heiner Geißler, »Immanuel Kant und der Weltfriede. In diesem Jahr könnte sich Kants Vision einer kritischen Weltöffentlichkeit als >negatives Surrogat< endlich erfüllen« in: Frankfurter Rundschau, 12. Februar 2004, S. 32. 
Entsprechung, als in seinem Völkerbundplan »ein friedliches Nebeneinander der Nationen « auch durch »das Prinzip der wirtschaftlichen Gleichberechtigung « weithin in Kombination mit freihändlerischen Grundsätzen (z.B. »Freiheit der Meere«, »offene Tür «, Meistbegünstigungsklausel) erreicht werden soll. ${ }^{99}$ Senghaas, der übermäßige »ökonomische Ungleichheit ... in sich modernisierenden und modernen Gesellschaften « als Hauptursache »politisch gewalttätiger Konflikte« diagnostiziert, sieht - anders noch als Erzberger - nicht mehr »die Staatenanarchie (samt Sicherheitsdilemma) « als das »Strukturmuster « zur Untersuchung bzw. Erklärung der heutigen Welt, sondern die global zu begreifende Entwicklungsproblematik. ${ }^{100}$ Als zeitgemäße Antwort genügt demnach »angesichts der krassen Asymmetrien in der Welt « nicht mehr »die klassische Position >Frieden durch Handek oder gar >Frieden durch Freihandel««, vielmehr plädiert er für eine »Politik aktiver Verteilungsgerechtigkeit « als »Mindestvoraussetzung für den sozialen Frieden in Gesellschaften wie zwischen ihnen. «101 Handelspolitisch ist nach Senghaas zur Forcierung »nachholender Entwicklung « im Interesse langfristigen Friedens eine situationsadäquate Mischung aus Abschottung und Öffnung gegenüber Weltmarkteinflüssen geboten. Insofern gelangt der Entwicklungsforscher an der Schwelle zum 21. Jahrhundert hinsichtlich dieser Frage in eine gewisse Nähe zu Erzbergers Position gegen Ende des Ersten Weltkriegs, als der Politiker sein grundsätzliches Plädoyer für eine liberale Handelspolitik mit dem Vorbehalt verband, dass »die Entscheidung über Schutzzoll und Freihandel « ein integrierender »Bestandteil der inneren staatlichen Selbständigkeit « bleiben müsse. ${ }^{102}$ Was Kant schon vor der eigentlich einsetzenden Industrialisierung sah, die wachsende Friedensrelevanz ökonomischer Faktoren, versuchte Erzberger vor dem gegebenen Hintergrund weltwirtschaftlicher Erfahrungen in sein Völkerbundkonzept zu integrieren; Senghaas hat überdies v.a. mit der für ihn typischen Brückenbildung zwischen Entwicklungs- sowie Friedens- und Konfliktforschung vielfältig konkretisiert wie theoretisch begründet, inwiefern sozio-ökonomische Entwicklung und Frieden in komplexen Zusammenhängen stehen.

\section{d) Weltbürgerliches Bewusstsein und Engagement als Befriedungsfaktoren}

Was schon bei Kant zwar als »negatives Surrogat « des »noch nicht vorhandenen Völkerbundes « eine Rolle spielt, nämlich »die Macht der öffentlichen Meinung der Weltbürger «103, wird bei Erzberger moralisch akzentuiert, wenn er davon spricht, die Völker würden anfangen »zu lernen, auch die realpolitische Macht der sittlichen Kräfte einzuschätzen «. ${ }^{104}$ In ibrer Entfaltung liegt ein Potenzial zur Forcierung kritischer Weltöffentlichkeit, um sie zur Kontrolle für die Mitwirkung an politischen

99 Vgl. Erzberger, Der Völkerbund. Der Weg zum Weltfrieden, aaO. (FN 2), S. 90-144, insbesondere Kapitel IX und X.

100 Vgl. Senghaas, Zum irdischen Frieden..., aaO. (FN 4), S. 12; außerdem Siegel, Ideen zur Friedensgestaltung..., aaO. (FN 3), S. $440 f$.

101 Senghaas, Zum irdischen Frieden..., aaO. (FN 4), S. 274.

102 Vgl. Siegel, Ideen zur Friedensgestaltung..., aaO. (FN 3), S. 416.

103 Geißler, »Immanuel Kant und der Weltfriede...«aaO. (FN 98), S. 32. 
Prozessen auf möglichst allen Ebenen bereit zu machen. Senghaas schließlich ergänzt diesen Aspekt globaler Befriedung als Lernprozess weltbürgerlichen Bewusstseins um eine praktische Komponente, indem er die sich damit erkennbaren Aufgaben und Vernetzungsoptionen auch für zivilgesellschaftliche Akteure konkreter benennt und ihre potenziellen Funktionen in »einer neuen Weltordnung « aufzeigt. ${ }^{105}$ Was Kant im dritten Definitivartikel der Friedensschrift zum darin vorskizzierten Weltbürgerrecht forderte, »daß die Rechtsverletzung an einem Platz der Erde an allen gefühlt« werden sollte, ${ }^{106}$ gewinnt heute noch größere Plausibilität. Zudem waren die Prämissen für die Einlösung dieser Forderung nie so günstig, wie im Zeitalter weltweiter Möglichkeiten medialer Echtzeit-Kommunikation. Sie im Sinne global wirksam werdender Mitverantwortung für eine weltbürgerliche Mobilisierung sittlicher Kräfte im Sinne Erzbergers zu nutzen und kombiniert mit den von Senghaas angeregten vieldimensionalen Lernprozessen zu qualifizieren, vermag erheblich näher an das Ziel dauerhaften Friedens heranzuführen. Hinzu kommt der Umstand, dass fortschreitende, wenn auch keineswegs flächendeckend identisch verlaufende »Globalisierung ... jenen Einbettungskontext, den Kant ... für die Idee des weltbürgerlichen Zustandes erörtert hatte, zu einer postnationalen Konstellation verdichtet « hat. ${ }^{107}$

\section{e) Schritte zum Frieden - inklusive UNO-Reform - mühsame Prozesse »wider Willen«}

Kants Vorstellung, durch eventuell notwendige Prozesse »wider Willen« über "eine weltbürgerliche Verfassung « zu dauerhaftem Frieden zu gelangen, ${ }^{108}$ ist auch bei Erzberger insofern präsent, als er die betreffende Stelle zitiert bzw. eine entsprechende Formulierung benutzt. ${ }^{109}$ In Senghaas' Werk zeigt sich die Bedeutung der zunächst paradox anmutenden Denkfigur sogar im Titel seines Buches zur interbzw. intrakulturellen Problematik Zivilisierung wider Willen. ${ }^{110}$ Zentral darin ist die Annahme prinzipieller Konfliktträchtigkeit von Modernisierungsprozessen, da nach Senghaas sich modernisierende Gesellschaften "mit sich selbst in Konflikt « geraten und die so geforderte "Kulturaufgabe immer auch wider die eigene Tradition « gerichtet ist. ${ }^{11}$ Nach wie vor dürften sich entscheidende Schritte zum Frieden als

104 Erzberger, Der Völkerbund. Der Weg zum Weltfrieden, aaO. (FN 2), S. 182 (Hervorhebung im Original).

105 Vgl. Senghaas, Zum irdischen Frieden..., aaO. (FN 4), S. 74-80 (insbesondere Unterpunkt 6.4 Akteure einer neuen Weltordnung).

106 Vgl. Kant, Zum ewigen Frieden..., aaO. (FN 5), S. 24 (Hervorhebung im Original).

107 Vgl. Habermas, Der gespaltene Westen, aaO. (FN 10), S. 74-80 sowie Bernhard Zangl / Michael Zürn, Frieden und Krieg. Sicherbeit in der nationalen und postnationalen Konstellation, Frankfurt am Main 2003.

108 Vgl. Kant, Über den Gemeinspruch, aaO. (FN 27), S. 64 (Hervorhebung im Original).

109 Vgl. Erzberger, Der Völkerbund. Der Weg zum Weltfrieden, aaO. (FN 2), S. 68 und Erzberger, Der Völkerbund als Friedensfrage, aaO. (FN 7), S. 4.

110 Dieter Senghaas, Zivilisierung wider Willen. Der Konflikt der Kulturen mit sich selbst. Frankfurt am Main 1998.

111 Vgl. ebd., S. 21 (Hervorhebung im Original). 
mühsame Prozesse »wider Willen« erweisen. Als Beispiel dafür kann die ebenso schwierige wie notwendige Aufgabe einer Reform und Stärkung der UNO angeführt werden. ${ }^{112}$ Überfällige Änderungen beim Sicherheitsrat, von seiner Zusammensetzung über Vetoverzicht bis zur Rechtspflicht zum Handeln, ${ }^{113}$ sind beharrlich gegen bisher Privilegierte durchzusetzen. Eine konsequentere Machtausübung der Weltorganisation mit den damit nötigen Souveränitätsabgaben von Staaten dürfte großteils nur widerwillig akzeptiert werden, solange weltbürgerliches Bewusstsein nur fragmentarisch entfaltet ist. Seine zureichende Weiterentwicklung - indirekt bereits von Erzberger mit Blick auf den Völkerbund gefordert ${ }^{114}$ - käme auch einer im Sinne erweiterter Partizipations- bzw. Legitimitäts- und Effizienzsteigerung bereiten Weltorganisation, damit ihrer Akzeptanz und Friedenswirksamkeit zugute. Die UNO hätte so auch als institutionelles »Rückgrat von global governance" ihre Zukunft. ${ }^{115}$

\section{Schlussbemerkung}

Die Beispiele der Bezüge von Konzepten Matthias Erzbergers und Dieter Senghaas' zur Friedensphilosophie Immanuel Kants zeigen auch erneut, wie dieser noch immer großteils prinzipielle Gültigkeit zukommt. Damit daraus aber auch noch für heute friedenswirksame Konsequenzen gezogen werden können, ist es unverzichtbar, die veränderten Rabmenbedingungen zu beachten. Obwohl bereits Erzbergerin Wahrnehmung des begonnenen sozialen Wandels - ähnlich wie später Senghaas akzentuiert eine zunehmende Politisierung in modernen Gesellschaften konstatiert hatte, ${ }^{116}$ sind die daraus ableitbaren Konsequenzen in Erzbergers Friedenskonzept noch nicht zureichend erkennbar; vielmehr hat er sich im Wesentlichen darauf beschränkt, Kants Völkerbundkonzept - gleichsam lediglich teilkontextuiert - in die Realität der Zeit nach dem Ersten Weltkrieg zu übertragen. Ähnliches gilt m. E. auch für andere damalige Konzepte eines Völkerbundes, nicht zuletzt für seine dann verwirklichte Version. Nach den Erfahrungen eines weiteren Weltkrieges und eines die Zivilisation bedrohenden atomar munitionierten Ost-West-Konfliktes geht es Senghaas darum, die Vision Kants für einen dauerhaften Frieden unter Be-

112 Zur prinzipiellen $>$ Reformagenda< siehe Habermas, Der gespaltene Westen..., aaO. (FN 10), S. 172-174.

113 Vgl. Senghaas, Zum irdischen Frieden..., aaO. (FN 4), S. 59, wo er fordert, dass der »Rechtscharakter der in der VN, insbesondere im Sicherheitsrat zu treffende Entscheidungen ... erstrangig sein « müssten; vertiefend hierzu Sabine von Schorlemer, »Imperative eines künftigen Friedensvölkerrechts" in: Dieter Senghaas (Hg.), Konstruktiver Pazifismus im 21. Jabrhundert. Symposium zum 80. Geburtstag von Dr. Gerald Mader, Berlin 2006, S. 33-66.

114 Vgl. Erzberger, Der Völkerbund. Der Weg zum Weltfrieden, aaO. (FN 2), S. 165, wo er beispielsweise betont, die Delegierten der Mitgliedstaaten sollten sich als »Träger des Völkerbundgedankens, des Friedens und des Ausgleichs« verstehen.

115 Vgl. Klaus Dieter Wolf, Die UNO. Geschichte - Aufgaben - Perspektiven, München 2005, S. 119.

116 Vgl. Siegel, Ideen zur Friedensgestaltung..., aaO. (FN 3), S. 393-396. 
rücksichtigung des industrialisierungsbedingten Wandels von traditionalen in moderne Gesellschaften auf dem Boden der vielfältig zerklüfteten Gegenwartswelt zu konkretisieren und jeweils kontextspezifische friedenspolitische Imperative zu benennen bzw. zu konzeptualisieren. Zu den offenen Fragen zählt dabei, inwieweit die mit der Industrialisierung seit dem Ende des 18. Jahrhunderts sich ebenfalls radikal wandelnden ökologischen Rahmenbedingungen schon hinreichend berücksichtigt sind, um ein zeitgemäßes Friedenskonzept für das 21. Jahrhundert zu bieten. Denn in ihm könnten sich Auswirkungen anthropogen verursachter oder verstärkter Umweltbelastungen mit Klimaveränderungen verdeutlichen, von denen Kant noch nichts ahnen konnte, die auch für Erzberger und seine Zeitgenossen nicht im Problemhorizont lagen. Heute jedoch wird absehbar, dass das mit einer weitgehend global wirksamen Industrialisierung sich möglicherweise dramatisch zuspitzende Ökologiedilemma von strukturbildender Qualität ${ }^{117}$ mit einer wobl auch friedenstheoretisch bedeutsamen Relevanz sein dürfte. ${ }^{118}$

117 Vgl. Senghaas, Wohin driftet die Welt?..., aaO. (FN 81), S. 154-161, wo der Autor ausdrücklich die Frage aufwirft: »Wird das ökologische Dilemma strukturbildend?«.

118 Vgl. z. B. Nicolas Richter, »Erderwärmung erstmals Thema im Sicherheitsrat. EU: Klimawandel gefährdet den Weltfrieden« in: Süddeutsche Zeitung, 18. April 2007 und Volker Teichert / Stefan Wilhelmy, »Der Wirtschaftsboom in China und Indien: Das Konfliktpotenzial der Umweltfolgen « in: Bruno Schoch / Andreas Heinemann-Grüder / Jochen Hippler / Markus Weingardt / Reinhard Mutz (Hg.), Friedensgutachten 2007, Berlin 2007, S. 232-244. 
Abb. 1: Synopse zu Analogien der Friedensschriften von Kant und Senghaas ${ }^{119}$

\begin{tabular}{|c|c|}
\hline Immanuel Kant & Dieter Senghaas \\
\hline $\begin{array}{l}\text { Zum ewigen Frieden } \\
\text { Ein philosophischer Entwurf }\end{array}$ & $\begin{array}{l}\text { Zum irdischen Frieden } \\
\text { Erkenntnisse und Vermutungen }\end{array}$ \\
\hline $\begin{array}{c}\text { Erster Abschnitt, } \\
\text { welcher die Präliminarartikel } \\
\text { zum ewigen Frieden unter Staaten enthält } \\
\text { (6 Postulate zum Abbau von Friedenshindernissen) }\end{array}$ & $\begin{array}{c}\text { Erster Abschnitt, } \\
\text { welcher Präliminarüberlegungen } \\
\text { zum irdischen Frieden enthält } \\
(» \text { para bellum } \ll)\end{array}$ \\
\hline \multirow[t]{3}{*}{$\begin{array}{c}\text { Zweiter Abschnitt, } \\
\text { welcher die Definitivartikel zum } \\
\text { ewigen Frieden unter Staaten enthält } \\
\text { 1. Definitivartikel (Rechtsstaat) } \\
\text { 2. Definitivartikel (Völkerbund) } \\
\text { 3. Definitivartikel (Weltbürgerrecht) }\end{array}$} & $\begin{array}{c}\text { Zweiter Abschnitt, } \\
\text { welcher die Definitivbestimmungen } \\
\text { zum irdischen Frieden entwickelt } \\
\text { (»para pacem«): } \\
\text { Wodurch und wie konstituiert } \\
\text { sich Frieden heute? } \\
\text { (v. a. zivilisatorisches Hexagon } \\
\text { und Weltordnungsproblematik) }\end{array}$ \\
\hline & $\begin{array}{l}\text { Dritter Abschnitt, } \\
\text { welcher friedenspolitische Vernunft } \\
\text { ermöglichende Bedingungen erläutert: } \\
\text { Wodurch und wie konstituiert sich } \\
\text { die Vernunft zum Frieden heute? }\end{array}$ \\
\hline & $\begin{array}{c}\text { Vierter Abschnitt, } \\
\text { welcher Kritiken zum »zivilisatorischen } \\
\text { Hexagon« kommentiert }\end{array}$ \\
\hline $\begin{array}{l}\text { Erster Zusatz: } \\
\text { Von der Garantie des ewigen Friedens }\end{array}$ & $\begin{array}{l}\text { Erster Zusatz: } \\
\text { Frieden - ein mehrfaches Komplex- } \\
\text { programm für dauerhaft gelingende } \\
\text { Vergemeinschaftung }\end{array}$ \\
\hline $\begin{array}{l}\text { Zweiter Zusatz: } \\
\text { Geheimer Artikel zum ewigen Frieden }\end{array}$ & $\begin{array}{c}\text { Zweiter Zusatz: } \\
\text { Friedenszonen - keine Chimäre }\end{array}$ \\
\hline $\begin{array}{c}\text { Anhang } \\
\text { - Über die Misshelligkeit zwischen } \\
\text { der Moral und der Politik, in Absicht } \\
\text { auf den ewigen Frieden } \\
\text { - Von der Einhelligkeit der Politik } \\
\text { mit der Moral nach dem } \\
\text { transzendentalen Begriffe } \\
\text { des öffentlichen Rechts }\end{array}$ & $\begin{array}{c}\text { Anhang } \\
\text { - Strukturbedingte Dilemmata der Welt } \\
\text { und Folgerungen für Friedenspolitik } \\
\text { - Interdependenzen im internationalen } \\
\text { System } \\
\text { - Die moderne Entwicklungsproblematik } \\
\text { und ihre Implikationen für Friedenspolitik } \\
\text { - Interkultureller Dialog angesichts } \\
\text { kultureller Globalisierung }\end{array}$ \\
\hline
\end{tabular}

119 Nach Grobgliederungen von Kant und Senghaas, Stichworte in Klammern ohne Anführungszeichen von A.S. 
Abbildung 2: Das zivilisatorische Hexagon von Dieter Senghaas ${ }^{120}$

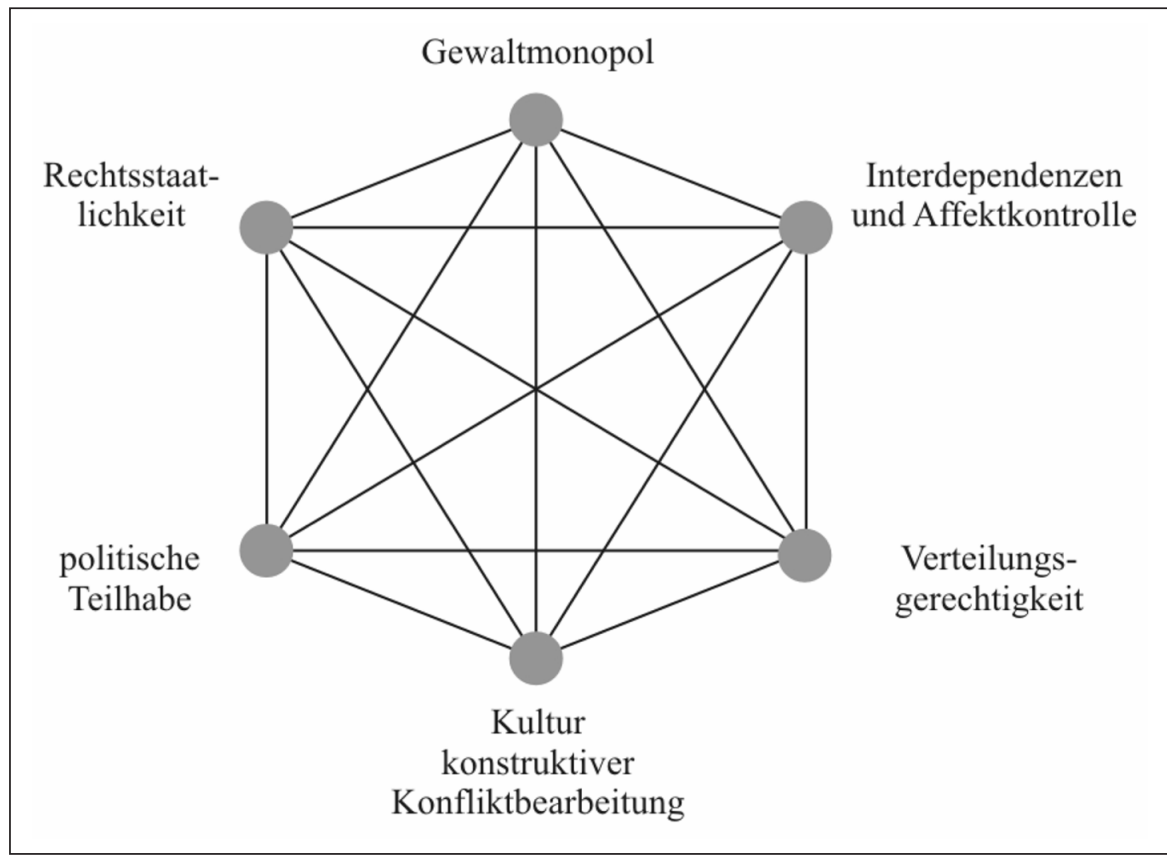

\section{Zusammenfassung}

1918 veröffentlichte der Politiker Matthias Erzberger sein berühmtes Buch Der Völkerbund. Der Weg zum Weltfrieden, das eine Vision zur Schaffung und Bewahrung eines globalen Friedens darstellt. Das Buch basiert explizit auf Ideen des deutschen Philosophen Immanuel Kant und beinhaltet eine Kritik des Militarismus. 2004 hat der renommierte Wissenschaftler und Friedensforscher Dieter Senghaas in seinem Buch Zum irdischen Frieden auf das Werk Immanuel Kants Bezug genommen und dessen Vision von 1795 Zum ewigen Frieden in die Ära nach dem Kalten Krieg übertragen.

Der Aufsatz illustriert Unterschiede und Ähnlichkeiten der Konzepte hinsichtlich ihrer Kantbezüge und betont die gemeinsamen Grundsätze für die Schaffung eines dauerhaften Friedens in der modernen Welt. Wie gezeigt, haben die Prinzipien von Kant noch immer Bedeutung für die Förderung einer friedlichen internationalen Gemeinschaft. Jedoch bedürfen seine Überlegungen einer Anpassung und Weiterentwicklung an eine sich stetig ändernde Umwelt wie sie von Senghaas und Erz-

120 Nach Senghaas, Zum irdischen Frieden..., aaO. (FN 4), S. 39. Für die wertvolle technische Unterstützung bei der Ausgestaltung des Aufsatzes danke ich Jürgen Baur. 
berger mehr oder weniger vorgenommen wurden. Mit einer derartigen Anpassung und Umsetzung - einschließlich einer Reform der UNO - könnten Konflikte mit mehr Aussicht auf Erfolg geregelt und dauerhafter Frieden weltweit wahrscheinlicher werden.

\section{Summary}

In 1918 the politician Matthias Erzberger published his famous book The League of Nations, the Way to the World's Peace which contains a roadmap for a global peace making and keeping concept. It is based explicitly on ideas of the German philosopher Immanuel Kant including a criticism of militarism. In 2004 the wellknown scientist and peace researcher Dieter Senghaas referred in his book Zum irdischen Frieden to the works of Immanuel Kant and transferred his vision of 1795 Zum ewigen Frieden into the post cold war era.

The article illustrates differences and similarities of the concepts of Erzberger and Senghaas concerning the reference to Kant's vision and stresses the common principles for the creation of a durable peace in a modern world. As shown the principles of Immanuel Kant still have relevance for the creation of a more peaceful international community. However, a further development is necessary in order to adapt the considerations to the changing international circumstances as done more or less by Senghaas and Erzberger. With such an adaption and implementation - including a reform of the UNO - conflicts could be tackled more successfully and the world might become more peaceful.

Alfons Siegel, The League of Nations, the Civilisatory Hexagon and the Future of the United Nations. References to Immanuel Kant in Peace Concepts of Matthias Erzberger and Dieter Senghaas

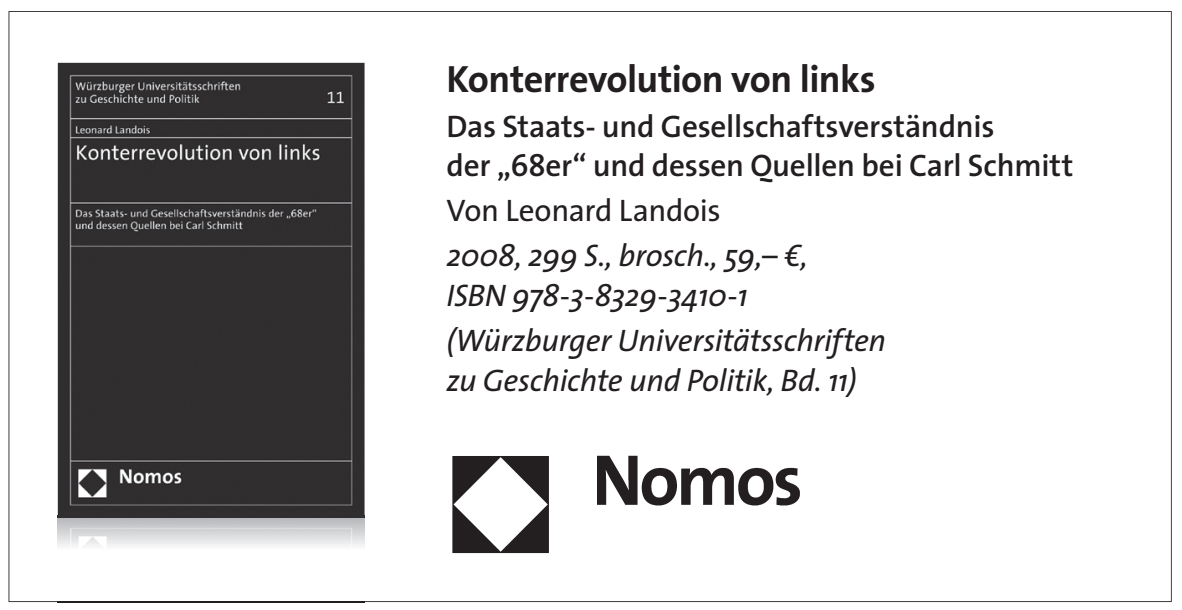

